5. Hanna Neumann, Varieties of groups, Ergebnisse der Mathematik ihrer Grenzgebiete, vol. 37, Springer-Verlag, Berlin, 1967.

6. Sheila Oates and M. B. Powell, Identical relations in finite groups, J. Algebra 1 (1964), 11-39.

The City University of New York and

The University of QUeEnsland, Brisbane, Australia

\title{
CONTINUITY OF THE VARISOLVENT CHEBYSHEV OPERATOR
}

\section{BY CHARLES B. DUNHAM}

Communicated by R. C. Buck, December 26, 1967

In this note we show that the Chebyshev operator $T$ is continuous at all functions whose best approximations are of maximum degree. Let $F$ be an approximating function unisolvent of variable degree on an interval $[\alpha, \beta]$ and let the maximum degree of $F$ be $n$. Let $P$ be the parameter space of $F$. All functions considered will be continuous and for such functions we define the norm

$$
\|g\|=\max \{|g(x)|: \alpha \leqq x \leqq \beta\} .
$$

The Chebyshev problem is, for a given continuous function $f$, to find an element $T(f)=F\left(A^{*}, \cdot\right), A^{*} \in P$, for which

$$
\rho(f)=\inf \{\|f-F(A, \cdot)\|: A \in P\}
$$

is attained. Such an element $T(f)$ is called a best Chebyshev approximation to $f$ on $[\alpha, \beta] . T(f)$ can fail to exist, but is unique and characterized by alternation if it exists. Definitions and theory are given in [1].

Lemma 1. Let $F(A, \cdot)$ be the best approximation to $f$ and $F$ have degree $n$ at $A$. Let $x_{0}, \cdots, x_{n}$ be an ordered set of points on which $f-F(A, \cdot)$ alternates $n$ times. If $\|f-g\|<\delta$ and $\|g-F(B, \cdot)\| \leqq \rho(g)+\delta$ then

$$
\begin{aligned}
(-1)^{i}\left[F\left(B, x_{i}\right)-F\left(A, x_{i}\right)\right] \operatorname{sgn}\left(f\left(x_{0}\right)-F\left(A, x_{0}\right)\right) & \geqq-3 \delta, \\
i & =0, \cdots, n .
\end{aligned}
$$

The lemma can be obtained using arguments similar to those of Rice [2, p. 63].

Lemma 2. Let $F$ be of degree $n$ (maximal) at $A$ then for given $\delta>0$ 
there exists $\eta(\delta)$ such that $\|F(A, \cdot)-F(B, \cdot)\|<\eta(\delta)$ if (1) holds and $\eta(\delta) \rightarrow 0$ as $\delta \rightarrow 0$.

The lemma is proven by arguments analogous to those of Tornheim cited after the next lemma.

Lemma 3. Let $F$ be unisolvent of degree $m$ at $A_{k}, k=0,1, \cdots$ and let $\left\{F\left(A_{k}, \cdot\right)\right\}$ converge pointwise to $F\left(A_{0}, \cdot\right)$ on $m$ distinct points then $\left\{F\left(A_{k}, \cdot\right)\right\}$ converges uniformly to $F\left(A_{0}, \cdot\right)$.

This result is a generalization of a result of Tornheim [2, pp. 72-73], [3, pp. 460-462] and is proven in the same way.

THEOREM. Let $F$ be unisolvent of variable degree. Let $f$ have a best approximation $F(A, \cdot)$ and $F$ be of degree $n$ (maximal) at $A$. There exists $\delta>0$ such that $\|f-g\|<\delta$ implies that $g$ has a best approximation. If $\left\{f_{k}\right\}$ converges uniformly to $f$ then $\left\{T\left(f_{k}\right)\right\}$ converges uniformly to $T(f)$.

Proof. Let $x_{0}, \cdots, x_{n}$ be as in Lemma 1. By definition of solvency of degree $n$ at $A$ there exists $\gamma>0$ such that if $\left|y_{k}-F\left(A, x_{k}\right)\right|<\gamma$, $k=1, \cdots, n$, then there exists a parameter $B$ satisfying

$$
F\left(B, x_{k}\right)=y_{k}, \quad k=1, \cdots, n .
$$

Using property $Z$ and maximality of $n$, it is easily seen that $F$ is unisolvent of degree $n$ at any such $B$, and hence $B$ is completely determined by (2). Choose $\delta$ such that $\eta(\delta)<\gamma / 2$ then by Lemmas 1 and 2 , if $\|f-g\|<\delta$ and $\|g-F(B, \cdot)\|<\rho(g)+\delta$, we have $\|F(A, \cdot)-F(B, \cdot)\|$ $<\gamma / 2$. Now let $\left\|g-F\left(B_{k}, \cdot\right)\right\|$ be a decreasing sequence with limit $\rho(g)$, then for all $k$ sufficiently large, $\left\|F(A, \cdot)-F\left(B_{k}, \cdot\right)\right\|<\gamma / 2$. The $n$-tuples of values at the points $x_{1}, \cdots, x_{n}$ of the approximants $F\left(B_{k}, \cdot\right)$ form therefore a bounded sequence with subsequence converging to an accumulation point $\left(y_{1}, \cdots, y_{n}\right)$, which determines a parameter $B$ at which $F$ is unisolvent of degree $n$. Using Lemma 3 we can show that for all $x \in[\alpha, \beta],|f(x)-F(B, x)| \leqq \rho(g)$ and so $F(B, \cdot)$ is a best approximation to $g$. The first part of the theorem is proven. Now let $\left\{f_{k}\right\}$ converge uniformly to $f$, then for all $k$ sufficiently large, $T\left(f_{k}\right)$ exists. From Lemmas 1 and 2 it follows immediately that $\left\|T(f)-T\left(f_{k}\right)\right\|$ converges to zero. The theorem is proven. From the arguments involving $n$-tuples we obtain

COROLlary. Let $F$ be unisolvent of variable degree, then the set of approximants of maximum degree is locally compact.

In developing the paper, no assumptions were made concerning the existence of $T(f)$. In case a unique best approximation exists to every 
continuous function, it is easily shown that if $f$ is an approximant, $\left\{f_{k}\right\}$ converging uniformly to $f$ implies that $\left\{T\left(f_{k}\right)\right\}$ converges uniformly to $f$, and the operator $T$ is continuous at every continuous function which is an approximant or has a best approximation of maximum degree. In the case of approximation by generalized rational functions it has been shown by Cheney and Loeb [4] that $T$ is continuous at no other continuous functions.

\section{REFERENCES}

1. John Rice, Tchebycheff approximations by functions unisolvent of variable degree, Trans. Amer. Math. Soc. 99 (1961), 298-302. 1964.

2. John Rice, Approximation of functions. Vol. 1, Addison-Wesley, Reading, Mass.,

3. L. Tornheim, On n-parameter families of functions and associated convex functions, Trans. Amer. Math. Soc. 69 (1950), 457-467.

4. E. W. Cheney and H. L. Loeb, On the continuity of rational approximation operators, Arch. Rational Mech. Anal. 21 (1966), 391-401.

University of Western Ontario, London, Canada 